\title{
Wi-Fi signal strength vs. magnetic fields for indoor positioning systems
}

\begin{abstract}
In this research we compare $\mathrm{Wi}-\mathrm{Fi}$ received signal strength indication and magnetic field based real-time location systems (RTLS) from various perspectives such as system complexity, accuracy and stability. To evaluate the performance of these systems we built several test fields with different types of environments. We will compare both approaches side-by-side and answer such issues as optimal calibration step (measurement interval), location accuracy, effect of minor and major environment changes to fingerprint DB and overall system accuracy.
\end{abstract}

Keyword: Indoor positioning; Wi-Fi; RSSI; Magnetic fields 